\title{
Flow simulations and validation for the first cohort of patients undergoing the Y-graft Fontan procedure
}

\author{
Weiguang Yang, PhD, ${ }^{\mathrm{a}}$ Frandics P. Chan, MD, PhD, ${ }^{\mathrm{b}}$ V. Mohan Reddy, MD, ${ }^{\mathrm{c}}$ Alison L. Marsden, PhD, ${ }^{\mathrm{d}}$ and \\ Jeffrey A. Feinstein, MD, MPH ${ }^{\mathrm{a}, \mathrm{e}}$
}

Objectives: In this study, with the use of computational fluid dynamics, we evaluate the postoperative hemodynamic performance of the first cohort of patients undergoing a handcrafted Y-graft Fontan procedure and validate simulation predictions of hepatic blood flow distribution against in vivo clinical data.

\begin{abstract}
Methods: An 18-12 $\times 2-\mathrm{mm}$ handcrafted Y-graft modification of the Fontan procedure was performed in 6 patients. Early (at the time of discharge) and 6-month postoperative 3-dimensional magnetic resonance imaging data were collected. Patient-specific models were constructed for flow simulations.
\end{abstract}

Results: Hepatic blood flow distribution varied among patients. Lung perfusion data $(n=3)$ showed good agreement with simulations. Postoperative asymmetry in hepatic blood flow distribution was reduced 6 months postoperatively. In 1 patient, low wall shear stress was found in the left limb of the Y-graft, corresponding to the location of subsequent thrombosis in the patient.

Conclusions: The credibility and accuracy of simulation-based predictions of postoperative hepatic flow distribution for the Fontan surgery have been validated by in vivo lung perfusion data. The performance of the Y-graft design is highly patient-specific. The anastomosis location is likely the most important factor influencing hepatic blood flow distribution. Although the development of thrombosis is multifactorial, the occurrence in 1 patient suggests that simulations should not solely consider the hepatic blood flow distribution but also aim to avoid low wall shear stress in the limbs. (J Thorac Cardiovasc Surg 2015;149:247-55)

See related commentary on pages 255-6.

The Fontan procedure, a surgical palliation used to treat patients with single ventricle heart defects, has evolved from the original atriopulmonary connection into the total cavopulmonary connection with 2 variants: the lateral tunnel and the extracardiac connection. ${ }^{1}$ In the extracardiac Fontan procedure, a polytetrafluoroethylene (Gore-Tex; WL Gore \& Associates Inc, Flagstaff, Ariz) conduit is used to connect the inferior vena cava (IVC) to the pulmonary artery (PA). ${ }^{1}$ A series of studies have demonstrated that geometry plays an important role in multiple hemodynamic parameters, leading to the belief that an optimized flow path could improve surgical outcomes. ${ }^{2-6}$ A computational fluid

\footnotetext{
From the Departments of Pediatrics (Cardiology), ${ }^{\mathrm{a}}$ Radiology, ${ }^{\mathrm{b}}$ Cardiothoracic Surgery, ${ }^{\mathrm{c}}$ and Bioengineering, ${ }^{\mathrm{e}}$ Stanford University, Palo Alto, Calif; and Department of Mechanical and Aerospace Engineering, ${ }^{\mathrm{d}}$ University of California San Diego, La Jolla, Calif.

This study was supported by the American Heart Association, a Burroughs Wellcome Fund Career Award at the Scientific Interface; a Leducq Foundation Network of Excellent Grant; a National Science Foundation CAREER Award No. OCI-1150184; and a University of California, San Diego Kaplan Fellowship. Disclosures: Authors have nothing to disclose with regard to commercial support. Received for publication March 26, 2014; revisions received Aug 19, 2014; accepted for publication Aug 23, 2014; available ahead of print Oct 14, 2014.

Address for reprints: Weiguang Yang, PhD, 750 Welch Rd, Suite 325, Palo Alto, CA 94304 (E-mail: wgyang@stanford.edu).

$0022-5223 / \$ 36.00$

Copyright (c) 2015 by The American Association for Thoracic Surgery http://dx.doi.org/10.1016/j.jtcvs.2014.08.069
}

dynamics (CFD) study by Dubini and colleagues ${ }^{2}$ suggested that an offset connection could reduce energy loss due to flow collision, leading to widespread adoption of the offset design by the surgical community. Two bifurcated extracardiac Fontan designs recently were proposed to replace traditional tube-shaped grafts based on CFD simulations. OptiFlo, proposed by Soerensen and colleagues, ${ }^{7}$ bifurcates both superior vena cava $(\mathrm{SVC})$ and IVC flow to the PAs, whereas the Y-graft by Marsden and colleagues $^{4,8}$ only replaces the tube graft with a bifurcated graft of IVC flow. Simulations on idealized and patientspecific models have shown that overall the Y-graft design improves energy loss, SVC pressure, and hepatic flow distribution (HFD), although no one-size-fits-all Y-graft design exists. ${ }^{4,9,10}$

On the basis of the previous simulation results, the Y-graft design has been translated into clinical use in 2 institutions. ${ }^{11,12}$ In a pilot study at Stanford, 6 patients underwent Y-graft extracardiac Fontan surgeries between June 2010 and March 2011. The technical success demonstrated the feasibility of the Y-graft; intermediate outcomes were reported in our accompanying study. ${ }^{12}$ Kanter and colleagues ${ }^{11}$ reported their preliminary results for using commercial off-the-shelf bifurcated grafts, showing that improved hemodynamic performance was achieved by distal anastomoses and that Y-graft cases had similar postoperative outcomes compared with traditional Fontan cases despite experiencing longer cardiopulmonary bypass times. 


$$
\begin{aligned}
& \text { Abbreviations and Acronyms } \\
& \text { CFD = computational fluid dynamics } \\
& \text { CT }=\text { computed tomography } \\
& \text { HFD = hepatic flow distribution } \\
& \text { IVC }=\text { inferior vena cava } \\
& \text { LPA }=\text { left pulmonary artery } \\
& \text { MRI }=\text { magnetic resonance imaging } \\
& \text { PA }=\text { pulmonary artery } \\
& \text { PAVM }=\text { pulmonary arteriovenous malformation } \\
& \text { RPA }=\text { right pulmonary artery } \\
& \text { SVC }=\text { superior vena cava } \\
& \text { WSS }=\text { wall shear stress }
\end{aligned}
$$

Because the previous hemodynamic evaluations of the Y-graft were based on virtual surgeries, ${ }^{4,10}$ in vivo validation for the computational modeling is needed. Therefore, this study has 2 main goals: (1) to evaluate the postoperative hemodynamic performance of the first cohort of patients undergoing the Y-graft Fontan and (2) to validate simulation predictions of HFD against lung perfusion data.

\section{MATERIALS AND METHODS}

\section{Surgical Technique and Clinical Data Acquisition}

The Y-graft implanted in 6 patients (YF1 to YF6) was custom-made for each patient by the surgeon with an 18-mm trunk and 12-mm limbs. The surgeon inspected the patient's anatomy in the operating room and handcrafted the Y-graft using his own judgment if technical feasibility was confirmed. Patients received standard postoperative care. Although the 6 surgeries were technically successful, the left limb of the Y-graft was found to be occluded in patient YF5 3 months after the Fontan procedure. Preoperative catheterization showed pulmonary arteriovenous malformations (PAVMs) in the right pulmonary artery (RPA). Early (at the time of discharge) and 6-month postoperative magnetic resonance imaging (MRI) scans were performed on patients YF1, YF2, and YF3. Pulmonary and vena cava flow were measured by phase-contrast MRI. For patients YF4 and YF6, only computed tomography (CT) images were collected. To quantify HFD in vivo, lung perfusion scans were performed on patients YF1 to YF3 in the early postoperative stage. $99 \mathrm{~m}$ Technetium-labeled macroaggregated albumin was administered intravenously in the patient's foot. The right-to-left perfusion ratio for lower body flow was then calculated by measuring the radioactivity in each lung field. ${ }^{13}$ We assume the hepatic blood flow is well mixed in the IVC before entering the lungs. Thus, the HFD is equal to the IVC flow distribution. Authors who performed flow simulations were blinded to lung perfusion data until all corresponding simulation results were reported. Patient YF5 underwent pre-Fontan and 3-month postoperative MRI scans. This pilot study was approved by the institutional review board, and informed consent was obtained. Readers are invited to read the accompanying article by Martin and colleagues ${ }^{12}$ for further clinical details.

\section{Model Construction}

After our previous work, patient-specific models were constructed using the SimVascular software package. ${ }^{14}$ Because thrombus developed in the left limb of the Y-graft of patient YF5, an additional virtual unblocked Y-graft was constructed for comparison. Patient-specific models and
MRI/CT images are shown in Figure 1. To examine the influence of graft size and anastomosis locations on wall shear stress (WSS) and HFD, 2 modified Y-graft designs were constructed for patient YF5. First, the right and left limb sizes were changed to $14 \mathrm{~mm}$ and $10 \mathrm{~mm}$, respectively (referred to as "R14-L10"). Then, model R12-L12 was created to have a more distal anastomosis and a more proximal anastomosis for the right and left limbs, respectively, without changing the size.

\section{Flow Simulation and Boundary Conditions}

To simulate blood flow, a 3-dimensional finite element Navier-Stokes solver was used with a Newtonian approximation for the viscosity and a rigid wall assumption. ${ }^{15}$ Pulsatile flow boundary conditions were applied to IVC and SVC inlets with a respiratory model following our previous work. ${ }^{16}$ For outlets, 3-element Windkessel models were tuned to achieve target Fontan pressures and pulmonary flow splits. ${ }^{10,17}$ A postoperative catheterization-derived Fontan pressure of $11 \mathrm{~mm} \mathrm{Hg}$ was used as the target for patient YF5, and $12 \mathrm{~mm} \mathrm{Hg}$ was assumed for all other patients because no catheterization data were available. For patients YF4 and YF6 who were imaged by CT, 3 pulmonary flow splits, $35 / 65,55 / 45$, and 75/25, were applied on the basis of lung perfusion studies by Cheng and colleagues ${ }^{13}$ and Seliem and colleagues. ${ }^{18}$ The IVC and SVC inflow conditions for these patients were taken from patient YF5 and scaled according to body surface area. ${ }^{16}$ In the current article, we use the format RPA/left pulmonary artery (LPA) to present pulmonary flow splits and HFD. Table 1 lists all patients' body surface area, blood flow rates, and pulmonary flow splits used in simulations.

To investigate the potential factors that led to the formation of thrombus in patient YF5, 3 scenarios were studied. We first simulated the flow environment at the time of the 3-month postoperative MRI after formation of the thrombus using the postoperative MRI data directly. Second, a complete Y-graft was virtually reconstructed by removing the limb blockage, and the same postoperative boundary conditions were used. Third, we simulated the presumed flow conditions in the early postoperative state using the unblocked Y-graft model with the preoperative pulmonary flow split, assuming that pulmonary remodeling progresses gradually and the pulmonary flow split does not change significantly within a short period after Fontan completion.

Lagrangian particle tracking was used to quantify HFD., 4 Approximately 10,000 particles were released at the IVC inlet every 1/ 100 cycle for a cycle. HFD was derived by computing the particle flux for particles traveling to the RPA and LPA, respectively. ${ }^{10}$

\section{RESULTS}

The results are divided into 3 parts. First, we present validation and longitudinal results for patients YF1, YF2, and YF3. Second, we present HFD under a range of pulmonary flow splits for patients YF4 and YF6. Third, we explore in detail the issue of thrombosis in patient YF5. Three different scenarios were simulated and compared to identify possible factors that triggered thrombus formation.

\section{Simulation Versus Lung Perfusion}

Figure 2, $A$, shows a comparison between simulation- and lung perfusion-derived HFD. The mean bias for simulationderived HFD is $4.3 \%$ (1.96 standard deviation $=10 \%)$. Good agreement was obtained for 3 patients, although the sample size was small. In patients YF1 and YF3, SVC flow blocked hepatic flow in the right and left limbs, respectively, because of a more proximal anastomosis. In 
YF1
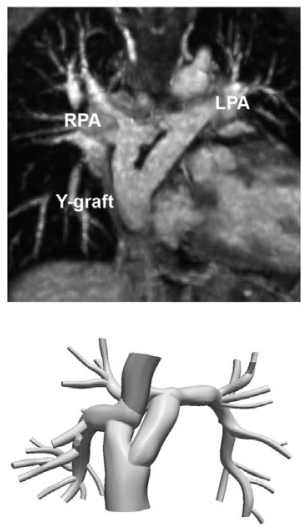

YF4
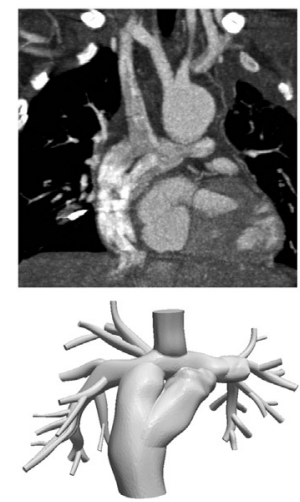

YF2
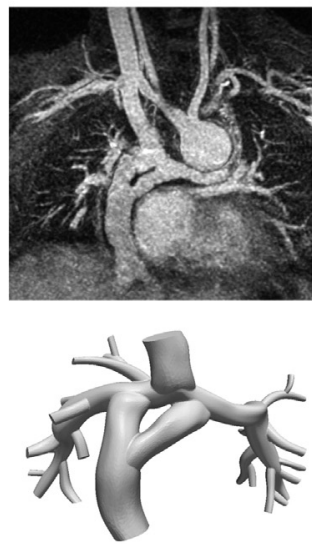

YF5
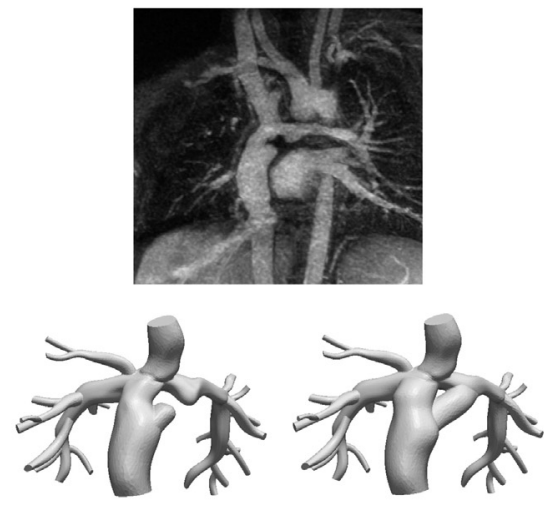

YF3
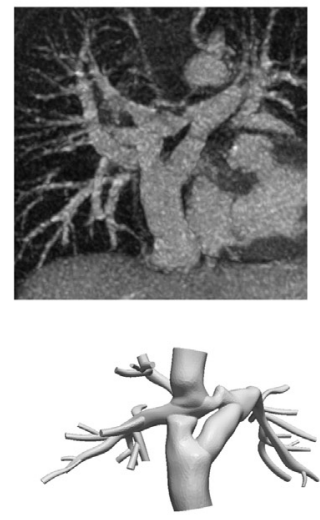

YF6
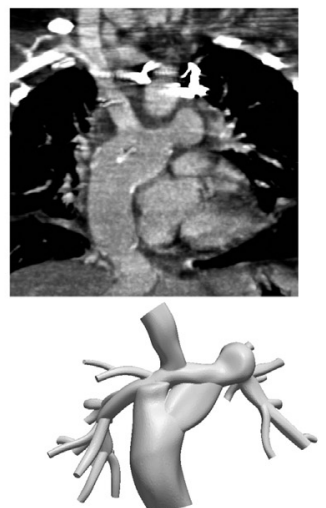

FIGURE 1. Postoperative MRI/CT images and models. Because thrombus developed in the left limb in patient YF5, an unblocked Y-graft was reconstructed for study. $L P A$, Left pulmonary artery; $R P A$, right pulmonary artery.

patient YF3, a distal anastomosis for the left limb that bypassed the stenosis in the LPA and a medial anastomosis for the right limb resulted in more than $60 \%$ of IVC flow streaming to the LPA despite the fact that the RPA received $60 \%$ of the total systemic venous flow.

\section{Longitudinal Hepatic Flow Distribution}

In Figure 2, $B$, HFD was computed by particle tracking and pulmonary flow splits were measured by MRI. Longitudinally, HFD in patients YF1 and YF3 became more even as the pulmonary flow split approached 50/50 and IVC flow

TABLE 1. Patients' flow conditions used in simulations

\begin{tabular}{lccccc}
\hline Data acquisition & Patients & BSA $\left(\mathbf{m}^{\mathbf{2}}\right)$ & IVC $(\mathbf{m L} / \mathbf{s})$ & SVC $(\mathbf{m L} / \mathbf{s})$ & Pulmonary flow split $(\%)$ \\
\hline Measured & YF1 & 0.59 & Early: 6.0 & Early: 14.0 & Early: $81 / 19$ \\
& & & 6 mo: 10.7 & 6 mo: 17.8 & 6 mo: $70 / 30$ \\
& YF2 & 0.62 & Early: 11.8 & Early: 15.0 & Early: $60 / 40$ \\
& & & 6 mo: 8.2 & 6 mo: 16.8 & 6 mo: $66 / 34$ \\
& YF3 & 0.56 & Early: 9.3 & Early: 9.5 & Early: $59 / 41$ \\
& & & 6 mo: 21.5 & 6 mo: 15.3 & 6 mo: $53 / 47$ \\
YF5 & 0.57 & Pre-Fontan: 11.5 & Pre-Fontan: 14.0 & Pre-Fontan: $81 / 19$ \\
Scaled & & & 3 mo: 11.7 & 3 mo: 12.2 & 3 mo: $54 / 46$ \\
& YF4 & 0.56 & 11.4 & 11.9 & $35 / 65,55 / 45,75 / 25$ \\
& YF6 & 0.55 & 11.2 & 11.7 & $35 / 65,55 / 45,75 / 25$ \\
\hline
\end{tabular}

The vena cava flow and pulmonary flow splits were measured by phase-contrast MRI except for patients YF4 and YF6, who had CT imaging. We use the format RPA/LPA to present pulmonary flow splits. For patients YF1, YF2, and YF3, "early" and "6 mo" denote measurements taken in the early (at the time of discharge) and 6-month postoperative stages, respectively. For patient YF5, preoperative and 3-month postoperative measurements were performed. BSA, Body surface area; IVC, inferior vena cava; SVC, superior vena cava. 

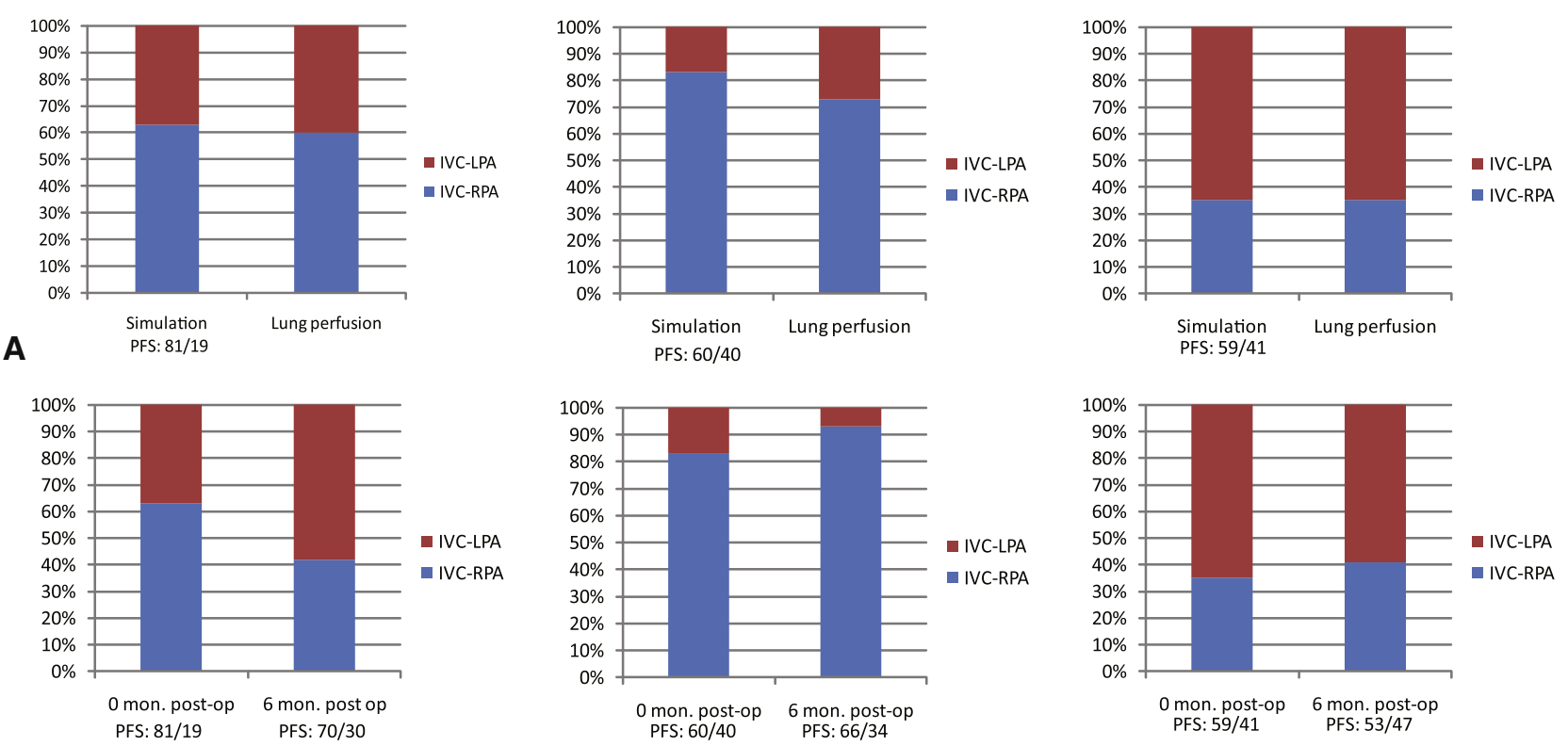

B

YF1

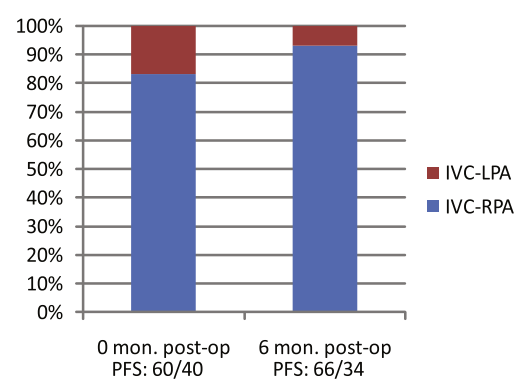

YF2

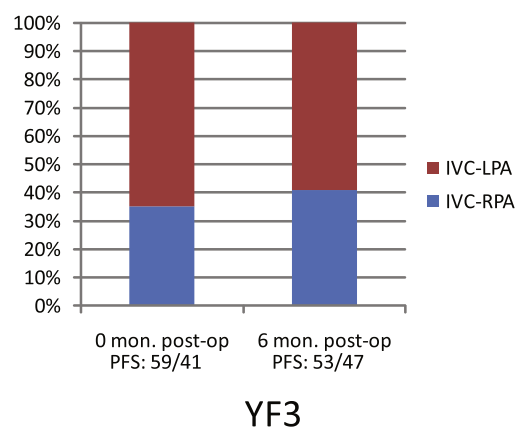

YF3

FIGURE 2. Hepatic flow distribution represented by percentage of inferior vena cava $(I V C)$ flow to the right pulmonary artery $(R P A)(I V C-R P A)$ and the left pulmonary artery $(L P A)(I V C-L P A)$. A, Comparison between early postoperative simulation-derived HFD and lung perfusion data for patients YF1, YF2, and YF3. B, Changes in HFD from the early to 6-month postoperative stages derived from simulations. PFS, Pulmonary flow split.

increased. In contrast, an opposite trend was found in patient YF2, but the changes in the pulmonary flow split and HFD were minor. The average change in HFD for these 3 patients is an $11 \%$ improvement (more balanced) and in the pulmonary flow split is $7.7 \%$.

\section{Hepatic Flow Distribution Estimation Without In Vivo Flow Conditions}

HFD was quantified under 3 assumed pulmonary flow splits of $35 / 65,55 / 45$, and $75 / 25$. In patient YF4, this resulted in percentages of hepatic flow streaming to the RPA of $55 \%, 69 \%$, and $88 \%$, respectively. In patient YF6, the results are $61 \%, 85 \%$, and $95 \%$, respectively.
RPA-skewed HFD was attributed to the blockage effect of SVC flow on the proximal anastomosis for the left limb.

\section{Thrombus Investigation}

Patient YF5's HFD under different flow conditions is summarized in Table 2 . In the early postoperative period, hepatic flow in patient YF5 was highly skewed to the RPA because of low resistance. Patient YF5's HFD was still skewed because of the occluded left limb even though the pulmonary flow split was found to be 54/46 3 months after the surgery. If the left limb remained patent, the HFD would be improved.

TABLE 2. Mean wall shear stress magnitude for Y-graft limbs and hepatic flow distribution

\begin{tabular}{|c|c|c|c|c|}
\hline Type & Patient (flow condition) & Left $\operatorname{limb}\left(\right.$ dynes $\left./ \mathrm{cm}^{2}\right)$ & Right limb (dynes $\left./ \mathrm{cm}^{2}\right)$ & HFD \\
\hline \multirow[t]{6}{*}{ Initial } & YF1 (early) & 5.0 & 10.8 & $63 / 37$ \\
\hline & YF2 (early) & 8.4 & 11.3 & $83 / 17$ \\
\hline & YF3 (early) & 4.2 & 5.4 & $35 / 65$ \\
\hline & YF 5 (early) & 4.6 & 25 & $88 / 12$ \\
\hline & YF 5 (3 mo) & 9.1 & 22 & 63/37 (unclotted) \\
\hline & & N/A & 19.7 & $82 / 18$ (clotted) \\
\hline \multirow[t]{4}{*}{ Modified } & YF5 R14-L10 (early) & 9.4 & 19.5 & $95 / 5$ \\
\hline & YF5 R14-L10 (3 mo) & 12.7 & 13.8 & $72 / 28$ \\
\hline & YF5 R12-L12 (early) & 11.7 & 13.2 & $99 / 1$ \\
\hline & YF5 R12-L12 (3 mo) & 9.9 & 8.1 & $85 / 15$ \\
\hline
\end{tabular}

Compared with other patients, patient YF5 had low WSS in the left limb in the early postoperative stage, but WSS in the left limb increased in the 3-month postoperative stage in which the pulmonary flow split changed from 81/19 to 54/46. Mean WSS for patient YF3 is low because of a lower cardiac output. The modified Y-grafts for patient YF5 increased mean WSS in the left limb in the early postoperative stage compared with the original Y-graft. All Y-graft designs for patient YF5 skewed hepatic flow to the RPA in the early postoperative stage, but HFD was improved in the 3-month postoperative stage. HFD, Hepatic flow distribution; N/A, not applicable. 


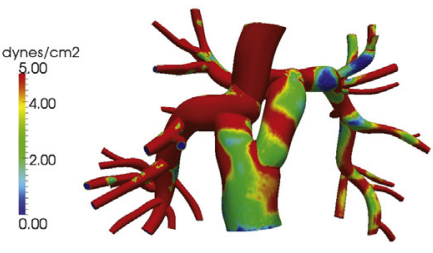

YF1

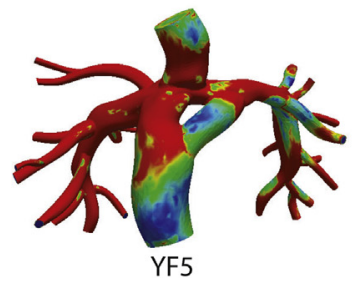

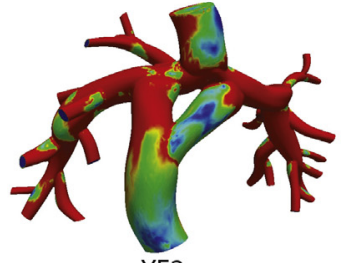

YF2

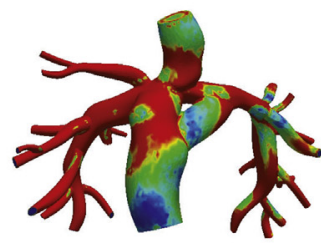

YF5 R14-L10

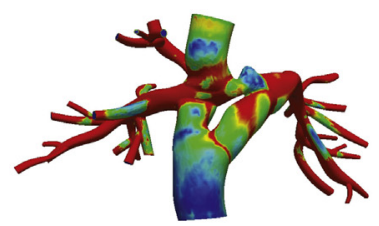

YF3

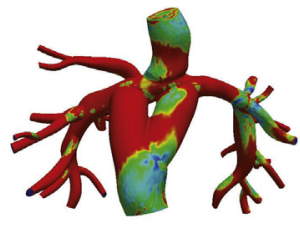

YF5 R12-L12

FIGURE 3. Time-averaged WSS magnitude for patients YF1, YF2, YF3, and YF5 in the early postoperative stage. YF5 R14-L10 and YF5 R12-L12 are 2 modified Y-graft designs for patient YF5. In the baseline model for patient YF5, a distal anastomosis for the left limb and a highly skewed pulmonary flow split resulted in a larger low WSS area in the left limb. In model R12-L12, WSS in the left limb was enhanced because of a proximal anastomosis that allowed SVC flow to wash the left limb.

Table 2 and Figure 3 show the time-averaged WSS magnitude of patients YF1, YF2, YF3, and YF5. For patient YF5, there is a persistent region of low WSS in the left limb for the early postoperative case. Overall, mean WSS is higher in the right limb than in the left limb. The WSS in the left limb increased in our simulation of the later time point when the pulmonary flow split was changed from $81 / 19$ to $54 / 46$. Although it is known that low WSS is
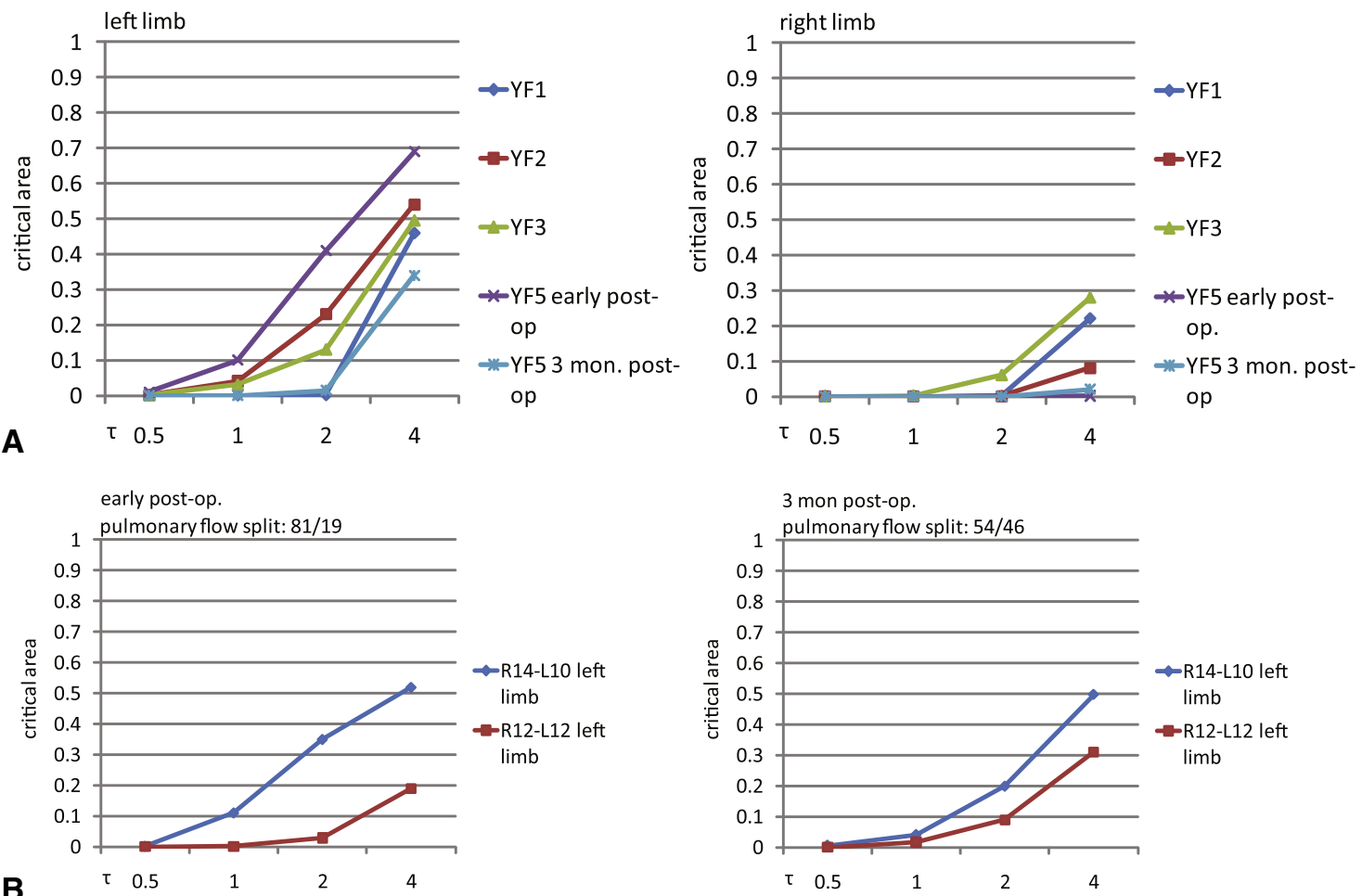

FIGURE 4. A, Percentage of low WSS area for 2 limbs in patients YF1, YF2, YF3, and YF5. For each threshold value $\tau\left(\right.$ dynes $\left./ \mathrm{cm}^{2}\right)$, the low WSS area relative to each limb surface was computed. B, Percentage of low WSS area for the left limb in patient YF5's modified designs. In model R12-L12, the low WSS area in the left limb can be effectively minimized by using a proximal anastomosis in which the SVC jet impinged the wall and the impact of the SVC jet on the WSS was reduced with increasing LPA flow. 
conducive to thrombosis formation, the exact threshold value remains unknown and is certain to vary among patients. ${ }^{20}$ Figure 4, A, shows the percentage area of low WSS with different threshold values for each limb in patients YF1, YF2, YF3, and YF5. Compared with the early postoperative results for patients YF1, YF2, and YF3, YF5's left limbs had a larger portion of low WSS for all threshold values used, although the mean WSS magnitude for the left limb in patient YF3 was also low because of a lower cardiac output. However, the percentage area of low WSS was significantly reduced in the 3-month postoperative stage.

Velocity fields (Figure 5) confirmed that an area of flow stagnation is evident in the left limb of patient YF5 under a pulmonary flow split of 81/19. In models YF4 and YF6 with HFD similar to YF5, fewer flow stagnation regions are observed because of the impingement of SVC flow.

Table 2 shows that mean WSS increased with decreasing graft size, and that the proximal anastomosis resulted in less low WSS area in the left limb because of the effect of the SVC jet. In Figure 4, B, model R12-L12 effectively minimized the low WSS area in the left limb by using a proximal anastomosis. Compared with the baseline model (Figure 4, A), model R14-L10 achieved a similar percentage of low WSS area for the left limb in the early postoperative stage for threshold values less than 2 dynes $/ \mathrm{cm}^{2}$.

\section{DISCUSSION}

In this study, we evaluated the hemodynamic performance for the first cohort of patients undergoing the Y-graft
Fontan. For the first time, we obtained MRI measurements at 2 time points, which directly compare the early and 6-month postoperative stages for Y-graft cases. Although only short-term data are available, measurements at 2 postoperative time points show that the ratios of caval flow and pulmonary flow split are not constant over time. Within 6 months after Fontan completion, a uniform increase of $33 \%$ was observed in SVC flow. IVC flow increased significantly by $105 \%$ in 2 patients, YF1 and YF3, but decreased by $31 \%$ in patient YF2. Although systemic venous flow increased in all patients, IVC flow was still less than SVC flow in all patients except YF3 at the 6-month postoperative time point. Likewise, Fogel and colleagues ${ }^{21}$ and Houlind and colleagues ${ }^{22}$ previously reported that the SVC contributed more flow to the venous return in patients receiving the lateral tunnel Fontan. This indicates that Y-graft cases likely follow the same trend in systemic venous flow distribution as other Fontan cases. At least 4 of 6 patients in this study had an RPA-predominant split, consistent with the measurements of Houlind and colleagues. ${ }^{22}$

In validating simulations against the lung perfusion data, good agreement was obtained in the 3 patients studied. The largest difference $(10 \%)$ between the simulation-derived HFD and lung perfusion in patient YF2 is still acceptable, considering potential sources of uncertainty in both simulation and clinical measurement. Although patient-specific data were incorporated into previous simulation studies, simulation-derived results had not been directly validated against clinical data in a blinded fashion in prior studies. The significance of this study is a validation that flow simulations correctly quantify patients' HFD. This provides a

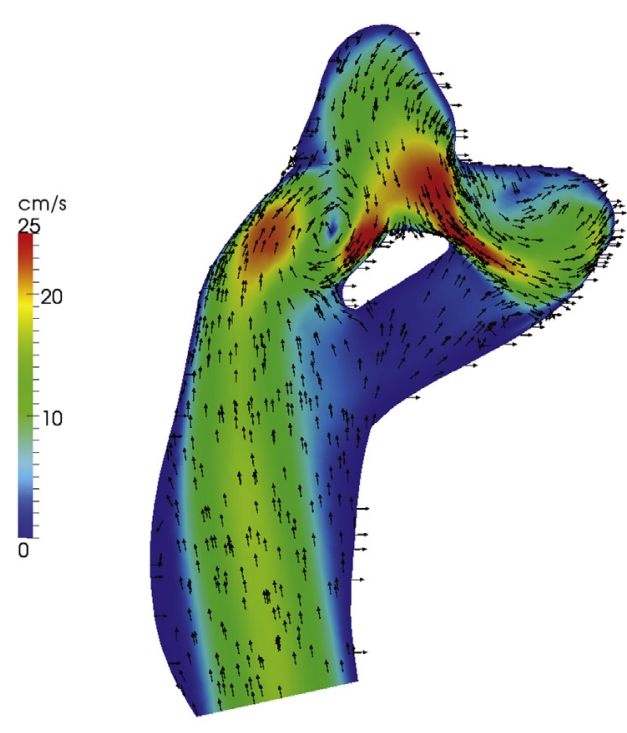

PFS: $81 / 19$

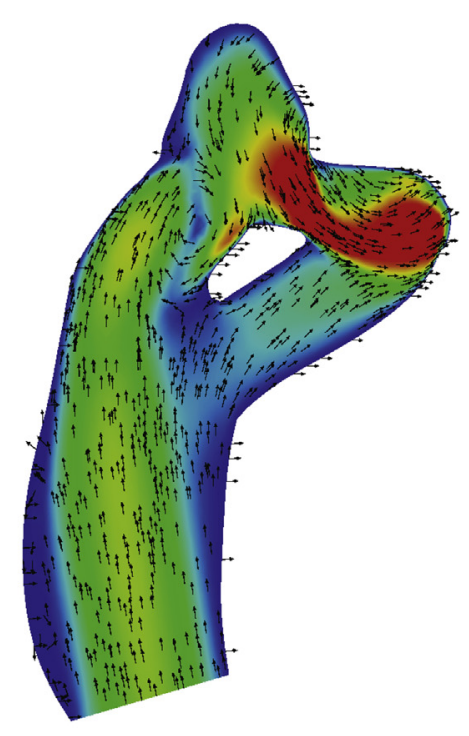

PFS: $54 / 46$

FIGURE 5. Velocity fields in the Y-graft for patient YF5 under 2 pulmonary flow splits. Flow stagnation is evident in the left limb under a pulmonary flow split of 81/19 that represents PAVMs in the right lung. In contrast, flow is increased and streamlined in the left limb under a pulmonary flow split of 54/45. PFS, Pulmonary flow split. 
basis for future predictive studies, albeit more patients should be used to achieve statistical significance.

Hepatic blood flow is known to carry a hepatic factor that inhibits PAVMs. ${ }^{23-25}$ Thus, HFD is a relevant issue to be considered in Fontan surgical design. HFD is largely influenced by the anastomosis locations and amount of SVC flow. In these Y-graft cases, the Y-graft was anastomosed to the PAs proximally relative to the SVC. For an uneven pulmonary flow split, a proximal anastomosis on the PA side with lower resistance is beneficial for reducing skewed hepatic flow. In patient YF1, the hepatic flow was relatively even despite a severely skewed pulmonary flow split, because the SVC jet blocked a part of hepatic flow streaming to the RPA. However, the same effect caused highly uneven HFD in patient YF2, who had a mildly uneven pulmonary flow split. These in vivo results were consistent with our previous virtual study. ${ }^{10}$ Anastomosing the left limb more distally could avoid the SVC flow impingement in the left limb in patients YF2, YF4, and YF6.

Because caval flow ratios and pulmonary resistance change with age, HFD is unlikely to remain constant over time. We found that HFD values shifted toward 50/50 over time in patients who had increased IVC flow and less skewed pulmonary flow splits. This agrees with our prior exercise simulation findings, ${ }^{10}$ such that increasing IVC flow could mitigate the adverse effects of high SVC flow and uneven pulmonary vascular resistance on HFD.

The preoperative and postoperative measurements in patient YF5 provide some interesting insight into pulmonary remodeling in patients with PAVMs. Patient YF5 had a preoperative pulmonary flow split of 81/19. It is well known that a lung with PAVMs typically has low pulmonary resistance due to a precapillary connection between the systemic and pulmonary venous return. ${ }^{6,23}$ At the 3-month postoperative examination, the pulmonary flow distribution was nearly even and PAVMs in the RPA had regressed. On the basis of the simulation results for patient YF5, we can infer that an uneven pulmonary flow split for patients with PAVMs before the Fontan procedure would likely change dramatically after surgery, and that streaming hepatic flow to the malformed lung reversed the PAVMs in this case, resulting in a less skewed pulmonary flow split.

For patients without PAVMs, the question of how the pulmonary flow split changes over time remains unclear and a long-term follow-up is needed. Our data suggest that pulmonary flow splits in patients without PAVMs are relatively stable within 6 months. Yin and colleagues ${ }^{26}$ reported a 5-year follow-up study on the pulmonary perfusion of 43 patients undergoing the Fontan. Their results showed that there are small differences $(3 \%$ and $2 \%$, respectively) in the pulmonary and IVC flow perfusion between the early postoperative and follow-up groups. ${ }^{26}$
However, perfusion data before the Fontan surgery were not available in their study.

The risk of thrombosis is another important issue that should be considered in addition to HFD. In patient YF5, thrombus was found in the left limb, although PAVMs in the right lung had regressed. Calculations with different threshold values indicate that low WSS and flow stasis in the left limb increased thrombotic risk and consequently led to complete occlusion of the left limb. Moreover, simulations with an unblocked Y-graft and a pulmonary flow split of 54/46 suggest that the thrombus likely formed before the PAVM regression that caused the pulmonary flow split to decrease from 81/19 to 54/46. Had PAVM regression occurred earlier, the increased WSS level in the left limb may have prevented thrombus formation. However, without further clinical evidence and data to support this timing, these findings remain speculative.

Although the presumed early development of thrombus in the left limb in patient YF5 may be driven by multiple factors, our findings suggest that low WSS and flow stasis are likely to be important causes. Thus, for patient YF5, the immediate surgical design goal should have been to distribute hepatic blood flow to the lung with PAVMs without introducing a large low WSS area, while at the same time keeping the long-term goal to achieve even HFD after PAVMs are regressed.

Compared with the original Y-graft for patient YF5, an $18-12 \times 2$ Y-graft with a proximal anastomosis for the left limb (YF5 R12-L12) minimized the low WSS area in the simulated early postoperative stage, although HFD was still skewed. However, HFD is later improved with increasing IVC flow. ${ }^{10}$ The unequal sized Y-graft (YF5 R14-L10) did not show substantial improvement in minimizing the low WSS area. Previous optimal designs considered a single objective solely. ${ }^{27}$ The formation of thrombus in patient YF5 showed that a single objective design without considering the dynamic process of pulmonary remodeling and thrombotic risk is inadequate. Our results suggest that the first 3 months post-Fontan may be a critical time period to prevent thrombosis in the Y-graft for patients with PAVMs, and that the risk likely decreases after PAVM regression. As pointed out in our previous studies, the superiority of the Y-graft cannot be guaranteed without a customized optimal design for each patient. In addition to optimizing the Y-graft geometry to minimize low WSS areas, more aggressive anticoagulation therapy may be needed for patients during the initial high-risk period. MRI and CFD could be used in these cases to monitor the WSS level in patients undergoing the Y-graft Fontan, providing extra information for their treatment.

Haggerty and colleagues ${ }^{28}$ recently reported postoperative simulation results for 5 patients undergoing the Y-graft Fontan at Children's Healthcare of Atlanta. In that study, the Y-graft design resulted in improved HFD but little 
difference in the connection resistance, compared with virtual T-junction and offset designs. These findings are likely due to the use of a smaller limb size $(9$ and $10 \mathrm{~mm}$ ) used for the Y-graft and more idealized graft geometry for the virtual tube-shaped models. Instead of custom Y-grafts, commercial bifurcated aortoiliac grafts were used in the Y-graft Fontan surgery in their study. ${ }^{28}$ Although the use of an "off the shelf" graft is consistent and well controlled in terms of fabrication, it is less flexible than the custom Y-graft in the choice of geometry, such as trunk-limb size and bifurcation angle. Because patients undergoing the Fontan have a variety of anatomic patterns, a custom graft may be more suitable for customized Fontan design.

\section{Study Limitations}

The follow-up time in this study was limited, and the number of study patients was relatively small. It is still too early to answer questions such as whether use of the Y-graft results in better mid-term or long-term outcomes than conventional designs. Longitudinal and serial data collection including the pre-Fontan stage should be performed on multiple patients in future studies.

Suture lines may contribute to the thrombus formation together with low shear stress. Intraoperator variability is inevitable, although all grafts were visually inspected by the surgeon. Because smooth walls were used in simulations, we were not able to evaluate the impact of suture lines on the thrombus formation. Current findings do not suffice to fully characterize the thrombus formation in patient YF5. Interaction between suture lines and blood flow should be examined in future studies.

Because of the lack of phase-contrast MRI data for patients YF4 and YF6, several assumptions were made for boundary conditions, increasing the uncertainty of our results. Thus, trends in HFD were presented instead of a single ratio. The prediction of postoperative pulmonary flow distribution from preoperative data should be addressed systematically by multiscale modeling in future studies. ${ }^{29}$ Rigid wall and Newtonian flow assumptions were used in this study. Long and colleagues ${ }^{30}$ showed that differences in HFD and energy efficiency due to wall compliance are small but that WSS was overpredicted by up to $17 \%$ in rest conditions by rigid wall simulations. ${ }^{31}$ Therefore, future accurate predictions of thrombotic risk may necessitate use of fluid structure interaction; however, the relative values used in this study may be adequate for risk assessment.

\section{CONCLUSIONS}

By comparing in vivo lung perfusion data, the accuracy of simulation-derived HFD was validated. Although the technical success of the Y-graft Fontan surgery demonstrated the feasibility of the Y-graft concept, overall more hepatic blood flow was channeled to the RPA in the early postoperative stage and HFD was improved as IVC flow increased and the pulmonary flow split became less unequal. A patient with unilateral PAVMs showed a significant change in his pulmonary flow split after Fontan completion because of the regression of PAVMs, whereas variations in other patients were less pronounced. Although sufficient hepatic blood flow was delivered to the malformed right lung to resolve PAVMs, thrombus likely developed in 1 limb with stagnant flow. Therefore, particular attention should be paid to WSS values and flow stasis in the early postoperative period to reduce the thrombotic risk in the Y-graft. This should be examined during the preoperative surgical planning phase, particularly for patients with existing PAVMs.

The authors thank Shawn Shadden, Irene Vignon-Clementel, Mahdi Esmaily Moghadam, and John Lamberti for expertise in numeric simulations and pediatric cardiac surgery, and Christina Ngo for assistance in model construction.

\section{References}

1. de Leval MR. The Fontan circulation: a challenge to William Harvey? Nat Clin Pract Cardiovasc Med. 2005;2:202-8.

2. Dubini G, de Leval MR, Pietrabissa R, Montevecchi FM, Fumero R. A numerical fluid mechanical study of repaired congenital heart defects. Application to the total cavopulmonary connection. J Biomech. 1996;29:111-21.

3. Bove EL, de Leval MR, Migliavacca F, Balossino R, Dubini G. Toward optimal hemodynamics: computer modeling of the Fontan circuit. Pediatr Cardiol. 2007; 28:477-81.

4. Marsden AL, Bernstein AJ, Reddy VM, Shadden SC, Spilker RL, Chan FP, et al. Evaluation of a novel Y-shaped extracardiac Fontan baffle using computational fluid dynamics. J Thorac Cardiovasc Surg. 2009;137:394-403.e2.

5. Marsden AL, Reddy VM, Shadden SC, Chan FP, Taylor CA, Feinstein JA. A new multi-parameter approach to computational simulation for Fontan assessment and redesign. Congenit Heart Dis. 2010;5:104-17.

6. De Zelicourt DA, Marsden AL, Fogel MA, Yoganathan AP. Imaging and patientspecific simulations for the Fontan surgery: current methodologies and clinical applications. Prog Pediatr Cardiol. 2010;30:31-44.

7. Soerensen DD, Pekkan K, de Zélicourt D, Sharma S, Kanter K, Fogel M, et al. Introduction of a new optimized total cavopulmonary connection. Ann Thorac Surg. 2007;83:2182-90.

8. Marsden AL, Vignon-Clementel IE, Chan F, Feinstein JA, Taylor CA. Effects of exercise and respiration on the hemodynamic efficiency of the total cavopulmonary connection. Ann Biomed Eng. 2007;35:250-63.

9. Yang W, Feinstein JA, Marsden AL. Constrained optimization of an idealized Yshaped baffle for the Fontan surgery at rest and exercise. Comput Meth Appl Mech Eng. 2010;199:2135-49.

10. Yang W, Vignon-Clementel IE, Troianowski G, Reddy VM, Feinstein JA, Marsden AL. Hepatic blood flow distribution and performance in conventional and novel Y-graft Fontan geometries: a case series computational fluid dynamics study. J Thorac Cardiovasc Surg. 2012;143:1086-97.

11. Kanter KR, Haggerty CM, Restrepo M, de Zelicourt DA, Rossignac J, Parks WJ, et al. Preliminary clinical experience with a bifurcated Y-graft Fontan procedurea feasibility study. J Thorac Cardiovasc Surg. 2012;144:383-9.

12. Martin MH, Feinstein JA, Chan FP, Marsden AL, Yang W, Reddy VM. Technical feasibility and intermediate outcomes of a hand-crafted, area-preserving, bifurcated "Y-graft" Fontan. J Thorac Cardiovasc Surg. 2015;149:249-55.

13. Cheng CP, Taur AS, Lee GS, Goris ML, Feinstein JA. Relative lung perfusion distribution in normal lung scans: observation and clinical implications. Congenit Heart Dis. 2006;1:210-6.

14. Schmidt JP, Delp SL, Sherman MA, Taylor CA, Pande VS, Altman RB. The Simbios National Center: Systems Biology in Motion. Proc IEEE Inst Electr Electron Eng. 2008;96:1266.

15. Taylor CA, Hughes TJR, Zarins CK. Finite element modeling of blood flow in arteries. Comput Methods Appl Mech Eng. 1998;158:155-96. 
16. Marsden AL, Vignon-Clementel IE, Chan FP, Feinstein JA, Taylor CA. Effects of exercise and respiration on hemodynamic efficiency in CFD simulations of the total cavopulmonary connection. Ann Biomed Eng. 2007;35: 250-63.

17. Troianowski G, Taylor CA, Feinstein JA, Vignon-Clementel I. Three-dimensional simulations in Glenn patients: clinically based boundary conditions, hemodynamic results and sensitivity to input data. J Biomech Eng. 2011;133: 111006.

18. Seliem MA, Murphy J, Vetter J, Heyman S, Norwood W. Lung perfusion patterns after bidirectional cavopulmonary anastomosis (hemi-Fontan procedure). $\mathrm{Pe}$ diatr Cardiol. 1997;18:191-6.

19. Shadden SC, Taylor CA. Characterization of coherent structures in the cardiovascular system. Ann Biomed Eng. 2008;36:1152-62.

20. Loth F, Fischer PF, Bassiouny HS. Blood flow in end-to-side anastomoses. Annu Rev Fluid Mech. 2008;40:367-93.

21. Fogel MA, Weinberg PM, Rychik J, Hubbard A, Jacobs M, Spray TL, et al. Caval contribution to flow in the branch pulmonary arteries of Fontan patients with a novel application of magnetic resonance presaturation pulse. Circulation. 1999;99:1215-21.

22. Houlind K, Stenbog E, Sorensen K, Emmertsen K, Hansen OK, Rybro L, et al. Pulmonary and caval flow dynamics after total cavopulmonary connection. Heart. 1999;81:67-72.

23. Duncan BW, Desai S. Pulmonary arteriovenous malformations after cavopulmonary anastomosis. Ann Thorac Surg. 2003;76:1759-66.
24. Pike NA, Vricella LA, Feinstein JA, Black MD, Reitz BA. Regression of severe pulmonary arteriovenous malformations after Fontan revision and "hepatic factor" rerouting. Ann Thorac Surg. 2004;78:697-9.

25. Imoto Y, Sese A, Joh K. Redirection of the hepatic venous flow for the treatment of pulmonary arteriovenous malformations after Fontan operation. Pediatr Cardiol. 2006;27:490-2.

26. Yin Z, Wang H, Wang Z, Zhu H, Zhang R, Hou M, et al. Radionuclide and angiographic assessment of pulmonary perfusion after Fontan procedure: comparative interim outcomes. Ann Thorac Surg. 2012;93:620-5.

27. Yang W, Feinstein JA, Shadden SC, Vignon-Clementel IE, Marsden AL. Optimization of a Y-graft design for improved hepatic flow distribution in the Fontan circulation. J Biomech Eng. 2013;135:011002.

28. Haggerty CM, Kanter KR, Restrepo M, de Zelicourt DA, Parks WJ, Rossignac J, et al. Simulating hemodynamics of the Fontan Y-graft based on patient-specific in vivo connections. $J$ Thorac Cardiovasc Surg. 2013;145:663-70.

29. Baretta A, Corsini C, Yang W, Vignon-Clementel IE, Marsden AL, Feinstein JA et al. Virtual surgeries in patients with congenital heart disease: a multi-scale modelling test case. Philos Trans A Math Phys Eng Sci. 2011;369:4316-30.

30. Long CC, Hsu M-C, Bazilevs Y, Feinstein JA, Marsden AL. Fluid-structure inter action simulations of the Fontan procedure using variable wall properties. Int $J$ Numer Method Biomed Eng. 2012;28:513-27.

31. Bazilevs Y, Hsu M-C, Besnon DJ, Sankaran S, Marsden AL. Computational fluid-structure interaction: methods and application to a total cavopulmonary connection. Comput Mech. 2008;45:77-89.

\title{
EDITORIAL COMMENTARY
}

\section{Optimizing hepatic flow distribution with the Fontan Y-graft: Lessons from computational simulations}

\author{
Elaine Tang, BEng, ${ }^{\mathrm{a}}$ and Ajit P. Yoganathan, $\mathrm{PhD}^{\mathrm{b}}$
}

See related article on pages 247-55.

Pulmonary arteriovenous malformations and thrombosis are 2 of the many long-term complications affecting patients with single-ventricle Fontan circulation. Pulmonary arteriovenous malformations have been related to the unbalanced distribution of hepatic nutrients to the 2 lungs. ${ }^{1,2}$ It has been shown that the flow phenomena in a blood vessel can be related to thrombus formation, and platelets preferentially adhere in low-shear zones. ${ }^{3}$ A bifurcated venous graft design, named "Optiflo," was proposed by

\footnotetext{
From the School of Chemical and Biomolecular Engineering, ${ }^{\text {a }}$ Georgia Institute of Technology, Atlanta, Ga; and The Wallace H. Coulter Department of Biomedical Engineering, ${ }^{\text {b }}$ Georgia Institute of Technology \& Emory University, Atlanta, Ga. Disclosures: Authors have nothing to disclose with regard to commercial support. Received for publication Sept 13, 2014; accepted for publication Sept 25, 2014; available ahead of print Oct 18, 2014.

Address for reprints: Ajit P. Yoganathan, PhD, Wallace H. Coulter Department of Biomedical Engineering, Georgia Institute of Technology and Emory University, Technology Enterprise Park, Suite 200, 387 Technology Circle, Atlanta, GA 30313-2412 (E-mail: ajit.yoganathan@bme.gatech.edu).

J Thorac Cardiovasc Surg 2015;149:255-6

$0022-5223 / \$ 36.00$

Copyright (c) 2015 by The American Association for Thoracic Surgery http://dx.doi.org/10.1016/j.jtcvs.2014.09.094
}

Soerensen and associates ${ }^{4}$ to optimize the energetics of the Fontan baffle and allow for a more even distribution of hepatic blood flow to the 2 lungs. One-half of the Optiflo results in the Y-graft connection (Figure 1). The first surgical cohort of patients with the Y-graft Fontan was presented by Kanter and coworkers, ${ }^{5}$ who demonstrated the surgical feasibility of this novel connection. These patients with Y-grafts were treated with an off-the-shelf iliac graft (manufactured by W.L. Gore \& Associates, Inc, Flagstaff, Ariz) and had similar postoperative courses to those of patients treated with the conventional total cavopulmonary connection. Postoperative flow modeling generally demonstrated a balanced distribution of inferior vena caval flow to both pulmonary arteries. ${ }^{5}$ In addition, Haggerty and colleagues ${ }^{6}$ demonstrated the similarities in the energetic performance of the Y-graft and the conventional total cavopulmonary connection by means of computational fluid dynamic studies.

"Flow simulations and validation for the first cohort of Y-graft Fontan patients" by Yang and colleagues ${ }^{7}$ in this issue of the Journal presents the simulation and validation of the first cohort of patients treated with custom-made Y-grafts, created by the surgeon in the operating room before implantation. This study also used patient-specific lung perfusion data to validate, for the first time, the hepatic 